\title{
Convergence of Digital TV Systems and Services
}

\author{
Maurizio Murroni, ${ }^{1}$ Sandro Scalise, ${ }^{2}$ Alessandro Vanelli-Coralli, ${ }^{3}$ \\ Sooyoung Kim, ${ }^{4}$ and Robert Briskman ${ }^{5}$
}

\author{
${ }^{1}$ DIEE, University of Cagliari, 09123 Cagliari, Italy \\ ${ }^{2}$ German Aerospace Center (DLR), Institute of Communications and Navigation, 82234 Wessling, Germany \\ ${ }^{3}$ DEIS/ARCES, University of Bologna, Viale Risorgimento 2, 40125 Bologna, Italy \\ ${ }^{4}$ College of Engineering, Chonbuk National University, Jeonju 561-756, South Korea \\ ${ }^{5}$ Sirius XM Radio Inc., USA
}

Correspondence should be addressed to Maurizio Murroni, murroni@diee.unica.it

Received 24 December 2009; Accepted 24 December 2009

Copyright (c) 2009 Maurizio Murroni et al. This is an open access article distributed under the Creative Commons Attribution License, which permits unrestricted use, distribution, and reproduction in any medium, provided the original work is properly cited.

The migration from analog to digital of the broadcasting technologies, already well consolidated for satellite systems, is becoming a reality also for terrestrial transmission. Digital Terrestrial Television (DTT) is also evolving to offer interactive services and a degree of flexibility which can be exploited to offer tailored applications to users which include, for instance interactivity, different levels of personalization, and innovative location-based, as well as context-aware, services. A clear example of this trend is given by the rising success of the Internet Protocol Television (IPTV) which allows for a degree of flexibility on offered services unknown to the traditional broadcasting systems. In this framework, several satellite operators are starting to launch IPTV services using direct satellite links, as well as some terrestrial internet service providers are offering digital TV channels embedded in the IP streaming over XDSL. Furthermore, IPTV services are likely to be broadcast also wirelessly, exploiting advanced broadband access technologies such as WiMAX, LTE, or LTEA. Last, but not least, TV and broadcast services for mobile users have also been deployed in many countries using DVB$\mathrm{H}$ and will be soon available on an even broader scale thanks to its satellite counterpart, DVB-SH. In the near future, a set of different technologies able to offer personalized and customized services to different classes of users are expected in the area of wireless broadcasting and convergence of technologies is auspicious. This concept entails different levels of convergence, namely, at terminal level (one device fits all), at service level (convergence of traditional fixed, mobile, and broadcast services), and at transport and network level with a common and standardized set of protocols and at access layer thanks to the harmonic coexistence of different radio technologies. This special issue aims to capture the state-of-the-art research work concerning the integration of DTT/Satellite/IPTV systems for the broadcasting of multimedia and interactive services.

There are very different directions in which researchers investigate new solutions in order to improve the quality of the rich media content delivered over various network types. In this context, this special issue illustrates some of these avenues and presents some of the most significant and promising results.

Papers have been grouped into four thematic sections.

The first section is devoted to Mobile Television and Interactive Multimedia Broadcasting Services. The first paper entitled "Converged digital TV services: the role of middleware and future directions of interactive television" provides a brief overview of digital TV converged services, to present and categorize the digital Television middleware technologies that contributed to it as well as possible future trends and directions. A new Television era of converged wireless and mobile content delivery, user-authored content, multimodal interaction, intelligent personalization, smart space awareness and 3D content sensations is foreseen, creating ambient and immersive experiences. The following one entitled "Interactive digital terrestrial television, the interoperability challenge in Brazil" introduces different standards implemented in existing Digital Terrestrial Television Broadcasting systems to allow the fruition of interactive 
services and applications through digital Set Top Boxes. It focuses on the interoperability issue between the Brazilian and the European architectures. Efforts in this area are reported from a Brazilian perspective including work to harmonize Brazilian and European standards. A wide variety of architectures, methods, and systems are reviewed to provide such applications, particularly for learning uses. In the third paper "Sofa-TV: the new digital landscape" the authors address the current state of channel offerings for the so-called Sofa-TV, that is, all digital television offers viewed through the "traditional" television screen. In this context, three digital platforms, Sat TV, DTT, and IPTV, are considered. After a description of the commercial offer, the authors identify the principal players and their strategies, in order to provide a few predictions as to the possible future changes in the industry. The analysis, carried out in the Italian context, has a general applicability and the conclusions apply to the general market.

The second section deals with Hybrid Cellular/NonCellular Broadcasting and contains three papers. The first one entitled "MBMS-IP multicast/broadcast in 3G Networks" extensively describes MBMS for 3GPP network, including the main features, architectural overview, and capabilities of MBMS added on various protocol stacks. As a use case, Mobile TV over MBMS in UMTS is addressed by using simulation results on capacity improvement by MBMS. MBMS evolution issues are also addressed. The second entitled "Video streaming transfer in a smart satellite mobile environment" concentrates on video streaming provided on buses that move in urban, suburban, or highway environments. A contents' source utilizes a satellite DVB-S2 link for transmitting video streams to a bus, which, in its turn, relays it to its passengers' devices. A bus works in a smart mode taking advantage of the knowledge of the exact points where it will not receive the satellite signal for a certain time period, due to fixed obstacles. Finally, the third one entitled "Modeling and performance analyses of hybrid cellular and broadcasting networks" considers the combination of the cellular network UMTS and the mobile broadcast network DVB-H, which form a hybrid network. The authors investigate the performance of hybrid networks and develop a system model, which describes the hybrid network and the load switching between both networks.

The third section addresses Source and Channel Coding Modulation and Signal Processing for Mobile Multimedia Broadcasting and it comprises four papers. The first one "Multiple description coding using data hiding and regions of interest for broadcasting applications" presents an innovative scheme for multiple description coding (MDC) with regions of interest (ROI) support to be adopted in high quality television. The scheme proposes to split the stream into two separate descriptors and to preserve the quality of the region of interest, even in case one descriptor is completely lost. To demonstrate its effectiveness, the algorithm has been implemented in two different scenarios, using the reference H.264/AVC codec and an MPEG framework to evaluate the performance in absence of motion-compensated frames on $720 \mathrm{p}$ video sequences. The next paper, entitled "Slow motion and zoom in HD digital videos using fractals" presents an alternative technique combining fractals theory and wavelet decomposition to achieve spatial zoom and slow motion replay of HD digital color video sequences. Fast scene change detection, active scene detection, wavelet subband analysis and, color fractal coding based on Earth Mover's Distance (EMD) measure are used to reduce computational load and to improve visual quality. In "AL-FEC for Improved mobile reception of MPEG-2 DVB-T transport streams," the authors investigate the use of application layer FEC protection in DVB-T (Digital Video Broadcasting-Terrestrial) networks for the provision of mobile services. The paper discusses some implementation aspects of AL-FEC in real scenarios and proposes an implementation based on Raptor codes and hash sequences. In the last work, entitled "The implementation of a 2/4/8 antennas configurable diversity OFDM receiver for mobile HDTV applications" a mobile OFDM receiver LSI is developed which includes two preFFT adaptive array (AA) antenna combiners and a post-FFT carrier diversity (CD) combiner. Using the LSI, several combinations of AA and CD diversity system can be configured as a trade-off approach to improve the performance of mobile ISDB-T receiver with reasonable system cost. The paper deals with the use of antenna diversity to improve the service availability for HDTV. Both simulation and measurement results are presented.

The last section covers System and Service for IPTV and Interactive $T V$. In the first paper "Theoretical models for video on demand services on peer-to-peer networks," the authors study the scenario P2P-based VoD services and propose four models of the peer behavior to evaluate the impact of the peer churn on the system performance. The models are compared by computing the resource that system has to add on top of the P2P network to satisfy all the download requests. Simulations show important relationships between playback buffer length, peer request rate, peer average lifetime, and server upload rate. The second paper, entitled "A software and hardware IPTV architecture for scalable DVB distribution," deals with the problem of mapping popular public broadcast services such as DVB or ATSC to IPTV with acceptable effort to IP-based television (IPTV). The authors propose a mapping using a light weight framework as an important step towards all-IP multimedia. Then, they present the Net-Ceiver architecture based on well-known standards such as IPv6, which allows zero configuration and high scalability. They also describe a low-cost FPGA implementation of the proposed NetCeiver architecture, which can concurrently stream services from up to six full transponders. The last paper is entitled "Concept for mobility and interconnections aspects in converged NGN based IPTV architecture" and discusses several potential architectures implementing the converged NGN IPTV concept. In this direction, ETSI TISPAN and ITU-T architecture for IPTV (IMS and non-IMS) are considered. In particular, an evolution of IMS-based IPTV where a single platform can serve multiple access networks is described along with several application scenarios. Last but not least, the paper "P2P and MPEG FGS encoding: a good recipe for multipoint video transmission in the internet" proposes a multipoint video broadcast framework over a heterogeneous 
content distribution P2P network. In the proposed system the source generated the video flow by using an MPEG4/FGS encoder, in such a way that no losses occur at the Baselayer stream even in the presence of short-term bandwidth fluctuations. Several solutions to this have been proposed but adoption has been hindered by many difficulties, particularly efficiency. Simulations of performance are presented which show that good efficiency would be achieved.

Maurizio Murroni Sandro Scalise Alessandro Vanelli-Coralli Sooyoung Kim Robert Briskman 

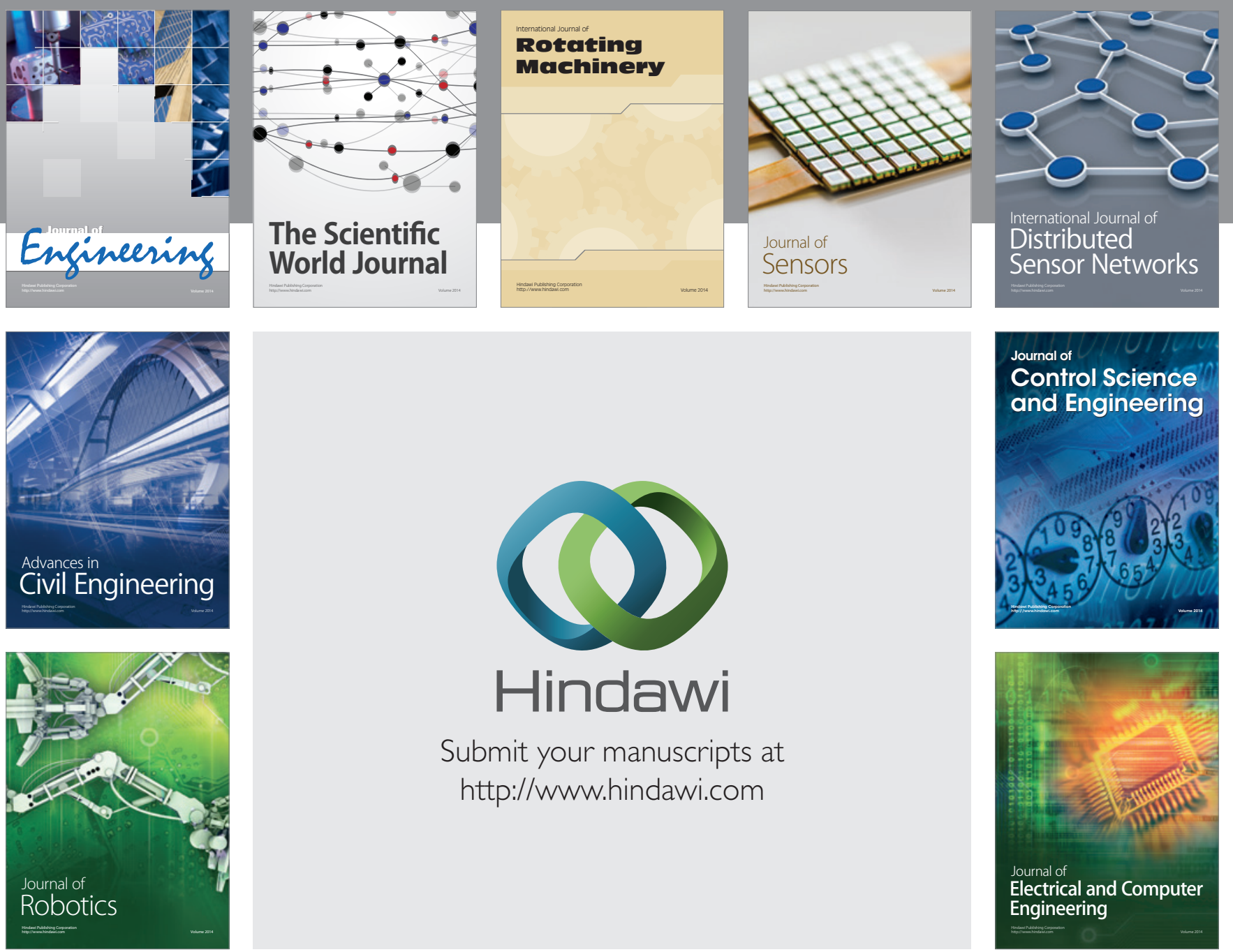

Submit your manuscripts at

http://www.hindawi.com
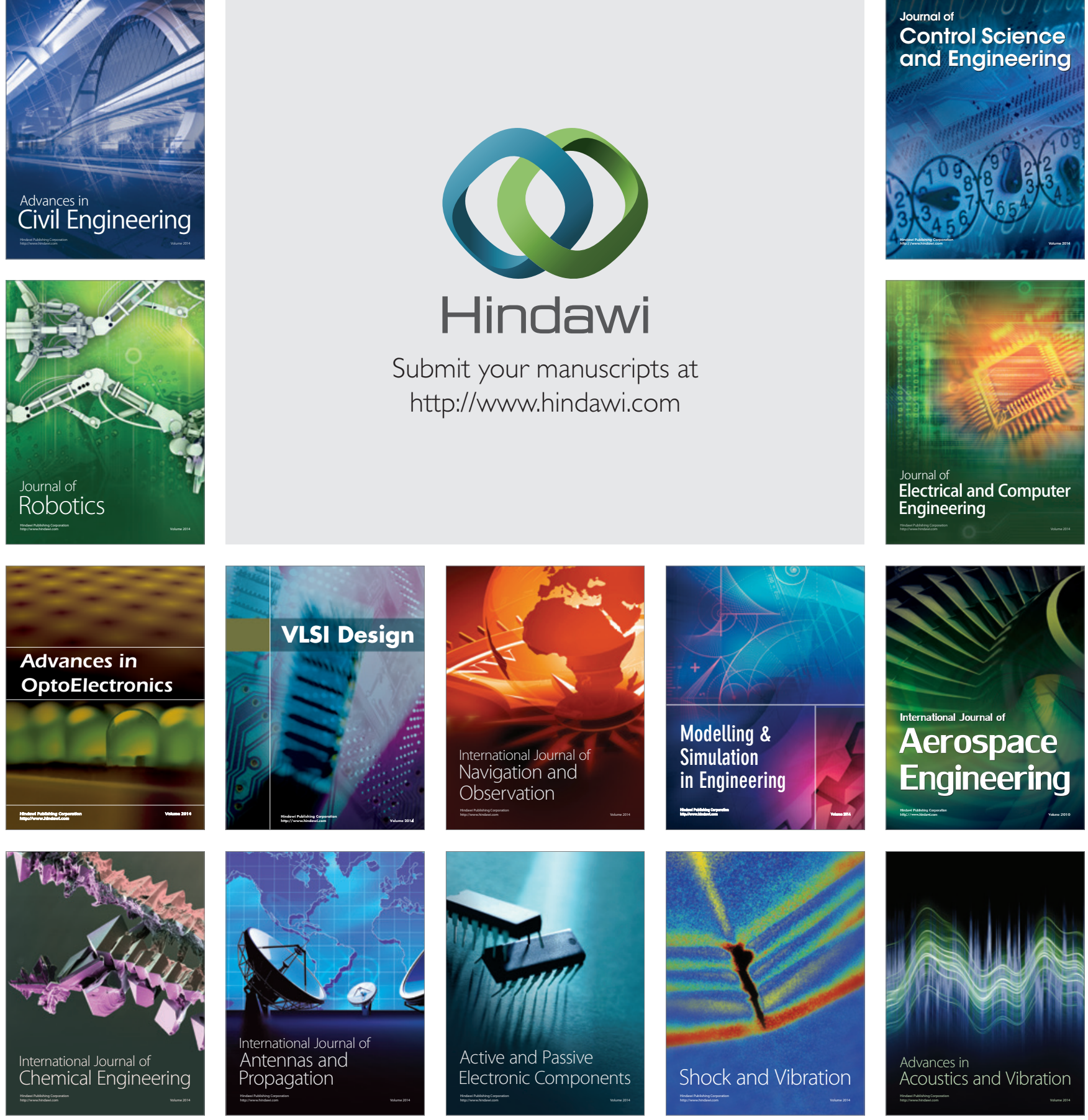